\title{
On the Solutions of $x_{n+1}=\frac{f\left(x_{n}\right)}{x_{n-1}}$ Where $f$ is Piecewise
}

\section{Linear}

\author{
M. B. Foreman, R. B. Holmes, W. E. Taylor* \\ Department of Mathematics, Texas Southern University, Houston, Texas
}

Received: May 18, 2015 / Accepted: July 12, 2016 / Published: September 25, 2016.

Abstract: Our goal in this paper is to examine the long-term behavior of solutions of the following difference equation $x_{n+1}=\frac{f\left(x_{n}\right)}{x_{n-1}}$, where $f$ is piecewise linear, and the initial values $x_{-1}$ and $x_{0}$ are non-zero real numbers. We examine the boundedness, periodicity, and the existence of oscillatory solutions.

Key words: nonlinear, difference equations, periodicity, oscillation

\section{Introduction}

In this paper, we are concerned with solutions of the difference equation

$$
x_{n+1}=\frac{f\left(x_{n}\right)}{x_{n-1}}
$$

Equation (1) has been studied in several works including papers by R. M. Abu-Saris, Saris and AI-Jubouri, B.D. Mestel and the book by Kulenovic and Ladas. In these works it was required that $f$ be positive and continuous on $(0, \infty)$. Generally these restrictions will not be necessary in this work. We do however require that $f$ be piecewise linear. One of our main concerns will be in determining how initial conditions effect periodicity as well as possible periods of solutions. There are cases where formulas for general solutions are obtained and solutions may not be periodic but have other interesting behavior.

Recall that a solution of (1) is periodic of period $\mathrm{p}$ if $x_{n+p}=x_{n}$ for all $\mathrm{n} \geq-1$ and is eventually periodic if there exists an integer $k$ such that $x_{n+p}=x_{n}$ for all $\mathrm{n}$ $\geq k$. Given a sequence $\left\{x_{n}\right\}_{n=-1}^{\infty}$, a subsequence of the form $\left\{x_{n}\right\}_{n=k}^{\infty}, \quad \mathrm{k}>-1, \quad$ is called an inductive

Corresponding author: W. E. Taylor, Department of Mathematics, Texas Southern University, Houston, Texas. E-mail: taylor_we@tsu.edu subsequence or a "tail" of the original sequence. It is clear that if $\left\{x_{n}\right\}$ is eventually periodic, then any tail is eventually periodic.

The initial values will be denoted as $x_{-1}=a, x_{0}=$ $b$, where $a, b \neq 0$.

The function $f$ will have one of the following forms

$$
\begin{aligned}
& \left(H_{1}\right): f(x)=\left\{\begin{array}{l}
A, x>0 \\
B, x<0
\end{array}\right. \\
& \left(H_{2}\right): f(x)=\left\{\begin{array}{l}
A x, x>0 \\
B, x<0
\end{array}\right. \\
& \left(H_{3}\right): f(x)=\left\{\begin{array}{l}
A x, x>0 \\
B x, x<0
\end{array}\right.
\end{aligned}
$$

where $\mathrm{A}$ and $\mathrm{B}$ are nonzero real numbers. The cases where $\mathrm{A}$ and $\mathrm{B}$ have the same sign or different signs will be investigated and the conditions $\left(H_{1}\right),\left(H_{2}\right)$ and $\left(H_{3}\right)$ are used to divide the main results.

\section{Results for}

$$
\left(H_{1}\right): f(x)=\left\{\begin{array}{l}
A, x>0 \\
B, x<0
\end{array}\right.
$$

Theorem 1.Assume $\mathrm{A}>0, \mathrm{~B}<0$

(i) Let $\left\{x_{n}\right\}$ be a solution of (1) such that $x_{-1}=a>0, x_{0}=b>0$. Then $\left\{x_{n}\right\}$ is periodic with period 4 and $x_{n}>0$ for all $n$. 
(ii) Let $\left\{x_{n}\right\}$ be a solution of (1) such that $x_{-1}=a<0$ or $x_{0}=b<0$, then $\left\{x_{n}\right\}$ is periodic with period 12.

Proof of (i): Let $\left\{x_{n}\right\}$ be a solution of (1).

Then $\left\{x_{n}\right\}=\left\{a, b, \frac{A}{a}, \frac{A}{b}, a, b, \ldots\right\}$. Thus $\left\{x_{n}\right\}$ is periodic with period 4 and all the terms are positive.

Proof of (ii): Let $\left\{x_{n}\right\}$ be a solution of (1) where $x_{-1}=a<0$ or $x_{0}=b<0$. We consider 3 cases,

Case 1. $\mathrm{a}<0<\mathrm{b}(-,+,-)$

$\left\{x_{n}\right\}$

$=\left\{a, b, \frac{A}{a}, \frac{B}{b}, \frac{a B}{A}, \frac{b A}{B}, \frac{A}{a}, \frac{B^{2}}{b A}, a, \frac{A b}{B}, \frac{B}{a}, \frac{B}{b}, a, b, \ldots\right\}$

Thus $\left\{x_{n}\right\}$ is periodic with period 12 .

Note that $x_{9}=\frac{B}{a}=\frac{B}{x_{-1}}$ and $x_{10}=\frac{B}{b}=\frac{B}{x_{0}}$.

Case 2. $\mathrm{a}<0, \mathrm{~b}<0(-,-,+)$

Since $x_{0}=a<0$ and $x_{1}=\frac{B}{a}>0$, we can apply

Case 1 to the sequence

$$
\begin{aligned}
x_{n}^{\prime}=x_{n+1}, \text { i.e. }, & \left\{x_{n}\right\}_{n=0}^{\infty}=\left\{x_{n}^{\prime}\right\} \\
= & \left\{b, \frac{B}{a}, \ldots, x_{11}^{\prime}=b, x_{12}^{\prime}=\frac{B}{a}, \ldots\right\}
\end{aligned}
$$

Note that $x_{11}=x_{10}^{\prime}=a$ and $x_{12}=x_{11}^{\prime}=b$ ensures that $\left\{x_{n}^{\prime}\right\}$ is periodic with period 12 .

Case 3. $a>0, b<0(+,-,-)$

Note that $x_{-1}=a, x_{0}=b, x_{1}=\frac{B}{a}<0$. Then the sequence

$$
\begin{aligned}
& \left\{x^{\prime \prime}\right\}_{n=-1}=\left\{x_{n+1}\right\} \\
& =\left\{b, \frac{B}{a}, \ldots, x^{\prime \prime}{ }_{11}=b, x^{\prime \prime}{ }_{12}=\frac{B}{a}, \ldots\right\}
\end{aligned}
$$

The reason for defining $\left\{x_{n}{ }_{n}\right\}$ as we did comes from the fact that the initial sign lineup for Case 3 is $(+,-,-)$ which resembles the Case 2 initial lineup $(-,-,+)$. Thus it follows that $\left\{x_{n}{ }_{n}\right\}$ is periodic with period 12 and the proof of the theorem is complete.

Theorem 2. Assume $\mathrm{A}<0, \mathrm{~B}>0$

(i) Let $\left\{x_{n}\right\}$ be a solution of (1) such that $x_{-1}=$ $a<0, x_{0}=b<0$. Then $\left\{x_{n}\right\}$ is periodic with period 4 and $x_{n}<0$ for all $n$.

(ii) Let $\left\{x_{n}\right\}$ be a solution of (1) such that $x_{-1}=a>0$ or $x_{0}=b>0$, then $\left\{x_{n}\right\}$ is periodic with period 12 .

Proof: Let $\left\{x_{n}\right\}$ be a solution of (1) and define a new sequence $\left\{y_{n}\right\}$ where $y_{n}=-x_{n}$ for each $n$. Then

$$
y_{n+1}=-x_{n+1}=-\frac{f\left(x_{n}\right)}{x_{n-1}}=\frac{f\left(x_{n}\right)}{-x_{n-1}}=\frac{f\left(-y_{n}\right)}{y_{n-1}}
$$

Consider the function $f_{1}(y)=f(-y)$.

Then

$$
\begin{gathered}
f_{1}(y)=f(-y)=\left\{\begin{array}{l}
A,-y>0 \\
B,-y<0
\end{array}=\left\{\begin{array}{l}
A, y<0 \\
B, y>0
\end{array}=\right.\right. \\
\left\{\begin{array}{l}
B, y>0 \\
A, y<0
\end{array}\right.
\end{gathered}
$$

Thus we may apply Theorem 1 to the sequence $\left\{y_{n}\right\}$ where the roles of $\mathrm{A}$ and $\mathrm{B}$ have been reversed. Note also that the initial conditions for $\left\{y_{n}\right\}$ are reversed, i.e., $x_{-1}, x_{0}<0$ yields $y_{-1}, y_{0}>0$ while $x_{-1}>$ 0 or $x_{0}>0$ becomes

$$
y_{-1}<0 \text { or } y_{0}<0 \text {. }
$$

Considering the cases where A and B have the same sign leads us to the following.

Theorem 3. Assume $\mathrm{A}>0$ and $\mathrm{B}>0$

Then every solution of (1) is periodic with period 4 . The proof of theorem 3 is easy and therefore omitted. However, it should be noted that when $x_{-1}, x_{0}>0$, all terms of the sequence have the same sign and when $x_{-1}, x_{0}<0$, all sequences are alternating.

The case where both A and B are negative yields a more interesting result.

Theorem 4. Assume $\mathrm{A}<0, \mathrm{~B}<0$

(i) Let $\left\{x_{n}\right\}$ be a solution of (1) such that $x_{-1}=a>0, x_{0}=b>0$, then

$$
\begin{aligned}
x_{4 n-1}=\left(\frac{B}{A}\right)^{n} a, & x_{4 n}=\left(\frac{A}{B}\right)^{n} b, x_{4 n+1} \\
= & \left(\frac{A}{B}\right)^{n}\left(\frac{A}{a}\right) \text { and } x_{4 n+2}=\left(\frac{B}{A}\right)^{n}\left(\frac{B}{b}\right) .
\end{aligned}
$$

(ii) Let $\left\{x_{n}\right\}$ be a solution of (1) such that $x_{-1}=a<0, x_{0}=b>0$, then 


$$
\begin{aligned}
& x_{4 n-1}=\left(\frac{B}{A}\right)^{n} a, x_{4 n}=\left(\frac{B}{A}\right)^{n} b, x_{4 n+1} \\
&=\left(\frac{A}{B}\right)^{n} \frac{A}{a} \text { and } x_{4 n+2}=\left(\frac{A}{B}\right)^{n} \frac{A}{b} .
\end{aligned}
$$

(iii) Let $\left\{x_{n}\right\}$ be a solution of (1) such that $x_{-1}=a<0, x_{0}=b<0$, then

$$
\begin{aligned}
x_{4 n-1}=\left(\frac{A}{B}\right)^{n} a & , x_{4 n}=\left(\frac{B}{A}\right)^{n} b, x_{4 n+1} \\
& =\left(\frac{B}{A}\right)^{n} \frac{B}{a} \text { and } x_{4 n+2}=\left(\frac{A}{B}\right)^{n} \frac{A}{b} .
\end{aligned}
$$

(iv) Let $\left\{x_{n}\right\}$ be a solution of (1) such that $x_{-1}=a>0, x_{0}=b<0$. Then

$$
\begin{aligned}
x_{4 n-1}=\left(\frac{A}{B}\right)^{n} a, & x_{4 n}=\left(\frac{A}{B}\right)^{n} b, x_{4 n+1} \\
& =\left(\frac{B}{A}\right)^{n} \frac{B}{a} \text { and } x_{4 n+2}=\left(\frac{B}{A}\right)^{n} \frac{B}{a} .
\end{aligned}
$$

Proof: We prove (i) by induction(ii) by using (i)on the subsequence $\left\{x_{n}\right\}_{n=0}^{\infty}$. Finally (iii) and (iv) are proved using the transformation $y_{n}=-x_{n}$ and applying (i) and (ii) to $\left\{y_{n}\right\}$. We now proceed with the proof of (i):

Let $\left\{x_{n}\right\}$ be a solution of (1) such that $x_{-1}=a>$ $0, x_{0}=b>0$. Let $P_{n}$ represent the statements

$$
\begin{aligned}
& x_{4 n-1}=\left(\frac{B}{A}\right)^{n} a, x_{4 n}=\left(\frac{A}{B}\right)^{n} b, x_{4 n+1} \\
&=\left(\frac{A}{B}\right)^{n} \frac{A}{a} \text { and } x_{4 n+2}=\left(\frac{B}{A}\right)^{n} \frac{B}{b}
\end{aligned}
$$

Note that $x_{-1}=a=\left(\frac{B}{A}\right)^{0} a, x_{0}=b=\left(\frac{A}{B}\right)^{0} b, x_{1}=$ $\frac{A}{a}=\left(\frac{A}{B}\right)^{0} \frac{A}{a}, x_{2}=\frac{B}{b}=\left(\frac{B}{A}\right)^{0} \frac{B}{b}$.

So $P_{0}$ is true. We now assume $P_{n}$ is true and prove $P_{n+1}$ is true.

Since $x_{4 n+2}=\left(\frac{B}{A}\right)^{n} \frac{B}{b}<0$, it follows that

$$
\begin{aligned}
x_{4(n+1)-1}=x_{4 n+3} & =\frac{B}{x_{4 n+1}}=\frac{B}{\left(\frac{A}{B}\right)^{n} \frac{A}{a}}=\left(\frac{B}{A}\right)^{n} \cdot \frac{B}{A} a \\
=\left(\frac{B}{A}\right)^{n+1} a & >0 .
\end{aligned}
$$

$$
\begin{aligned}
& x_{4(n+1)}=x_{4 n+4}=\frac{A}{x_{4 n+2}}=\frac{A}{\left(\frac{B}{A}\right)^{n} \frac{B}{b}}=\left(\frac{A}{B}\right)^{n} \cdot \frac{A}{B} b \\
& =\left(\frac{A}{B}\right)^{n+1} \quad b>0 \\
& x_{4(n+1)+1}=x_{4 n+5}=\frac{A}{x_{4 n+3}}=\frac{A}{\left(\frac{B}{A}\right)^{n+1} a}=\left(\frac{A}{B}\right)^{n+1} \frac{A}{a} \\
& <0 \text {. } \\
& x_{4(n+1)+2}=x_{4 n+6}=\frac{B}{x_{4 n+4}}=\frac{B}{\left(\frac{A}{B}\right)^{n+1} b}=\left(\frac{B}{A}\right)^{n+1} \frac{B}{b} \text {. }
\end{aligned}
$$

Thus $P_{n+1}$ is true when we assume $P_{n}$ to be true. Using the principle of mathematical induction it follows that $P_{n}$ holds for $n=0,1,2, \ldots$ parts (ii), (iii), (iv) of our theorem can also be proven by induction, however we will use an alternative method.

Proof of (ii). We consider the case where $x_{-1}=a<0$ and $x_{0}=b>0$. Define the sequence $\left\{x_{n}^{\prime}\right\}_{n=-1}^{\infty}=\left\{x_{n+1}\right\}_{n+1=0}^{\infty}$. Thus $x_{n}^{\prime}=x_{n+1}$. We then apply (i)to the sequence $x_{n}^{\prime}$, noting that $a^{\prime}=x_{-1}^{\prime}=$ $x_{0}=b$ and $b^{\prime}=x_{0}^{\prime}=x_{1}=\frac{A}{a}$,

$$
\begin{gathered}
x_{4 n-1}=x_{4 n-2}^{\prime}=x_{4(n-1)+2}^{\prime}=\left(\frac{B}{A}\right)^{n-1} \frac{B}{b^{\prime}} \\
=\left(\frac{B}{A}\right)^{n-1} \cdot \frac{\frac{B}{A}}{a}=\left(\frac{B}{A}\right)^{n} a \\
x_{4 n}=x_{4 n-1}^{\prime}=\left(\frac{B}{A}\right)^{n} a^{\prime}=\left(\frac{B}{A}\right)^{n} b \\
x_{4 n+1}=x_{4 n}^{\prime}=\left(\frac{A}{B}\right)^{n} b^{\prime}=\left(\frac{A}{B}\right)^{n} \frac{A}{a} \\
x_{4 n+2}=x_{4 n+1}^{\prime}=\left(\frac{A}{B}\right)^{n} \frac{A}{a^{\prime}}=\left(\frac{A}{B}\right)^{n} \frac{A}{b}
\end{gathered}
$$

Hence (ii) holds and follows from part (i). Finally by using the transformation $y_{n}=-x_{n}$ and making the appropriate changes in the roles of $\mathrm{A}$ and $\mathrm{B}$ we get

$$
\begin{aligned}
y_{4 n-1}=\left(\frac{B}{A}\right)^{n} y_{-1}, y_{4 n}=\left(\frac{A}{B}\right)^{n} y_{0}, y_{4 n+1} \\
=\left(\frac{A}{B}\right)^{n} \frac{A}{y_{-1}}, y_{4 n+2}=\left(\frac{B}{A}\right)^{n} \frac{B}{y_{0}}
\end{aligned}
$$


from which (iii) will follow. Part (iv) is done in a similar manner.

Corollary 1. When $\mathrm{A}<0, \mathrm{~B}<0$ and $\mathrm{A} \neq \mathrm{B}$, the solutions of (1) are not periodic.

Corollary 2. When $\mathrm{A}<0, \mathrm{~B}<0$ and $\mathrm{A} \neq \mathrm{B}$, the solutions of (1) are unbounded and oscillatory.

\section{Results for}

$$
\left(H_{2}\right): f(x)=\left\{\begin{array}{c}
A x, x>0 \\
B, x<0
\end{array}\right.
$$

Theorem 1.Assume $\mathrm{A}>0, \mathrm{~B}<0$

(i) Let $\left\{x_{n}\right\}$ be a solution of (1) such that $x_{-1}=a>0, x_{0}=b>0$. Then $\left\{x_{n}\right\}$ is periodic with period 6 and $x_{n}>0$ for all $n$.

(ii) $\operatorname{Let}\left\{x_{n}\right\}$ be a solution of (1) such that either $x_{-1}=a<0$ or $x_{0}=b<0$. Then $\left\{x_{n}\right\}$ is periodic with period 9.

Proof of (i): Assume $\left\{x_{n}\right\}$ is a solutions of (1) with $a, b>0$.

Then

$$
\left\{x_{n}\right\}=\left\{a, b, \frac{A b}{a}, \frac{A^{2}}{a}, \frac{A^{2}}{b}, \frac{A a}{b}, a, b, \ldots\right\} .
$$

So $\left\{x_{n}\right\}$ is a 6-cycle of positive terms

Proof of (ii): We consider 3 cases for (ii)

Case $1 x_{-1}=a<0, x_{0}=b>0$. Then

$$
\left\{x_{n}\right\}=\left\{a, b, \frac{A b}{a}, \frac{B}{b}, \frac{B a}{A b}, a, \frac{A b}{a}, \frac{B}{a}, \frac{B}{b}, a, b, \ldots\right\}
$$

So $\left\{x_{n}\right\}$ is a 9-cycle. Note that $x_{6}=\frac{B}{a}$ and $x_{7}=\frac{B}{b}$.

Case $2 x_{-1}=a<0, x_{0}=b<0, x_{1}=\frac{B}{a}>0$

Since the initial sign lineup $(-,-,+)$ resembles the initial sign lineup for Case 1 , we let $x_{n}^{\prime}=x_{n+1}$ for $n=-1,0,1,2, \ldots$ So $\left\{x_{n}^{\prime}\right\}$ behaves like a tail of $\left\{x_{n}\right\}$, i.e, $\left\{x_{n}^{\prime}\right\}$ is a 9-cycle, indeed, $\left\{x_{n}^{\prime}\right\}=\left\{b, \frac{B}{a}, \frac{B}{b}, \ldots, x_{8}^{\prime}=b, x_{9}^{\prime}=\frac{B}{a}, \ldots\right\}$. To see that $\left\{x_{n}^{\prime}\right\}$ is a true 9- cycle, simply note that $x_{7}^{\prime}=\frac{B}{b^{\prime}}=$
$\frac{B}{x_{0}^{\prime}}=\frac{B}{x_{1}}=a$

$\underline{\text { Case } 3} x_{-1}=a>0, x_{0}=b<0, x_{1}=\frac{B}{a}<0$

Since $x_{0}<0, x_{1}<0$, the sequence $\left\{x_{n}\right\}_{n=0}^{\infty}=$ $\left\{x_{0}, x_{1}, x_{2}, \ldots\right\}$ satisfies the conditions for Case 2 and is therefore a 9-cycle.

\section{Results for}

$$
\left(H_{3}\right): f(x)=\left\{\begin{array}{l}
A x, x>0 \\
B x, x<0
\end{array}\right.
$$

Theorem 1. Assume $\mathrm{A}>0, \mathrm{~B}<0$

Then every solution of (1) is periodic with period 6 .

Proof: We consider only two cases, $(i) x_{-1}=a>$ $0, x_{0}=b>0$ and (ii) $x_{-1}=a>0, x_{0}=b<0$. As shown earlier in this work, the remaining cases can be reduced to (i) and (ii) using the transformation $y_{n}=-x_{n}$.

We proceed to prove (i).

Note that

$$
\left\{x_{n}\right\}=\left\{a, b, \frac{A b}{a}, \frac{A^{2}}{a}, \frac{A^{2}}{b}, \frac{A a}{b}, a, b, \ldots\right\}
$$

$\operatorname{Thus}\left\{x_{n}\right\}$ is a 6-cycle consisting of positive terms.

For the proof of (ii) note that

$$
\left\{x_{n}\right\}=\left\{a, b, \frac{B b}{a}, \frac{A B}{a}, \frac{A B}{b}, \frac{A a}{b}, a, b, \ldots\right\}
$$

We see that $\left\{x_{n}\right\}$ is an alternating 6-cycle.

Theorem 2. Assume $\mathrm{A}<0, \mathrm{~B}>0$

Then every solution of (1) is a 12-cycle. The proof of Theorem 2 is straightforward and uses techniques previously done in the work. For this reason the proof is omitted.

\section{References}

[1] Abu-Saris, R.M., Characterization of rational periodic sequences, Journal of Difference Equations and Applications 2000, Vol. 6, pp. 233-242.

[2] Mestel, B.D., On globally Periodic Solutions of the difference equation $x_{n+1}=\frac{f\left(x_{n}\right)}{x_{n-1}}$, Journal of Difference Equations and Applications 2003, Vol. 9(2), pp.201-209. 